\title{
Confidentiality and serious harm in genetics
}

\author{
European Journal of Human Genetics (2005) 13, 399. doi:10.1038/sj.ejhg.5201349 \\ Published online 12 January 2005
}

I am pleased that Lucassen and Parker $^{1}$ have raised the serious issue of respect for confidentiality in the context of genetic information in your journal. There have been calls to reassess the medical profession's commitment to confidentiality when harm may come to those family members from whom relevant information has been withheld. When should practitioner's duty to other family members override their wish to respect the privacy of their patient?

While I agree with their conclusions, I do think that the two cases they describe could have been managed differently - perhaps with fewer resulting difficulties. In the first case (Case 1), the problem would either have been resolved or made explicit by the standard clinical practice of seeking access to the medical records of the affected individual. The genetic counselling team could have asked their client - the daughter of Jim and Mary - to obtain her father's consent (if he were still competent) for the genetics team to gain access to his medical records for the purpose of genetic counselling. Consent would either be granted or denied - in either case, the situation is much clearer and the question of a different diagnosis of Jim's dementia would be up for discussion.

In Case 2, the difficulties arise in part - at least, the problems take the shape they do - because of prior decisions made by the genetic service providers about access to molecular testing for susceptibility to breast cancer. The decision only to perform molecular testing on at-risk individuals when their family's mutation is known has been made because this ensures that those tests performed provide the most information possible. This is justified - I suspect - by an appeal to cost-benefit ratios and the efficient allocation and use of limited resources but this denies any sort of service to those whose family structure is not maximally helpful. Testing the at-risk niece Mrs L, who is seeking a prophylactic mastectomy because of her increased risk of breast cancer, would not be a maximally efficient use of resources when viewed from the public health perspective but it would be very useful in either providing strong reassurance or in justifying an important clinical decision. Performing the test might actually be more useful than Mrs L realises, but that is not a reason for not carrying out the test. Lucassen and Parker should not let the perfect (allocation of resources) be the enemy of the good (clinical practice). Furthermore, and without breaking Mrs L's confidence, the genetics team could arrange to discuss with Miss D her wish for privacy, in the hope that she might be persuaded to allow at least her mutation result to be used for the benefit of others in the family. Perhaps this could be achieved in a carefully negotiated and only semianonymous manner. Whether or not that consent could be obtained, however, the testing could go ahead on Mrs L if the genetics team agreed to less restrictive criteria for testing. The ethical issue in this case, I contend, is largely a product of clinical decisions that relate to rationing and the maximally efficient use of resources.

In both cases, I argue that a resolution of the ethical difficulties is largely in the hands of the clinicians - and if they choose not to resolve the issues then that is what requires a careful ethical analysis.

Angus Clarke

Department of Medical Genetics, University of Wales College of Medicine, Cardiff CF14 4XN, Wales, UK. e-mail: ClarkeAJ@Cardiff.ac.uk

\footnotetext{
Reference

1 Lucassen A, Parker M: Confidentiality and serious harm in genetics-preserving the confidentiality of one patient and preventing harm to relatives. EJHG 2004; 12: 93-97.
}

\section{Dilemma still not resolved}

European Journal of Human Genetics (2005) 13, 399-400. doi:10.1038/sj.ejhg.5201350

Published online 12 January 2005

Reply to the Letter from A Clarke

We would like to thank Angus Clarke for his insightful comments on our paper ${ }^{1}$ but disagree with some of the conclusions he draws.
In cases such as that of Jim and Mary, we agree that the standard policy is to seek access to the medical records of the affected person. Indeed, such consent was sought when the daughter was seen in the department (space 
constrictions in the article prevented us from describing all the details of the cases). However, despite the best efforts of the clinicians involved, no consent was ever obtained, and so the dilemma remained. Were we to leave the daughter in the dark or go against Jim's expressed wishes? The clinicians felt uneasy about telling the daughter that they could not give her specific risk information without further details about Jim, when in fact they had these details readily available. The clinicians were aware that the daughter was at a quite different risk from the one she thought she was at, but felt unable to tell her without his consent. Our conclusion is that while such cases will be rare and clinical staff can, and should, try to facilitate communication between family members, in some families this will not be successful. It is therefore important to debate how such cases ought to be dealt with when they do arise.

We also agree with Clarke that much genetic testing is currently influenced by resource allocation issues. However, contrary to what he suggests, in our department and in others, we do, in fact, offer genetic testing to unaffected individuals if a search for the particular familial mutation in affected relatives is not possible. We counsel such women that while the finding of a mutation is likely to be informative, the absence of a mutation cannot distinguish between a true negative (Mrs L has not inherited the familial inherited tendency that is present) and a false negative (Mrs L has inherited it, but the test is not yet good enough to find it). Since testing for mutations in families with a strong history of breast and/or ovarian cancer is currently at best $70 \%$ sensitive, a 'negative' result holds at least a 30\% chance of being a false negative. This is because the BRCA genes are large and current testing techniques do not cover the genes comprehensively and because BRCA 1 and 2 gene mutations do not explain all family histories such as that of Mrs L and Miss D. Even private genetic testing in the US (which is therefore free from resource allocation issues) admits to at least $15 \%$ of BRCA1 and 2 tests being falsely negative; it does not quantify the percentage of family histories such as those of Mrs L that are likely to be due to non-BRCA1/2 genes: Its overall sensitivity may, therefore, well be similar to $70 \%$ quoted above (http://www.myriadtests.com/ provider/doc/tech_specs_brac.pdf accessed 20 November 2004).

In our case, Mrs L was not prepared to tolerate the uncertainty of a 'negative' result and wanted a prophylactic mastectomy unless we could prove that she had not inherited the familial tendency. The problem in this case is of course partly a temporal one. Had we known about the existence of Miss D and her result before Mrs L was counselled, a certain degree of fudging (with its own attendant ethical problems) might have been possible. However, since we had already explained the uncertainty of a negative genetic test result, this could not be changed without breaching Miss D's confidentiality. Furthermore, standard laboratory practice would be to use Miss D's sample as a positive control for Mrs L's predictive test, and thus her sample would have to be used without her consent.

Again, in response to Clarke, the genetics team had discussed the benefit of using Miss D's result for her wider family with her, but after an initial meeting Miss D had declined any further contact with the team.

As we have suggested, such cases represent a very small fraction of the caseload of clinical genetics departments, most families are happy to share their information, and where not, clinicians can often help by encouraging and facilitating communication. Nevertheless, the question remains, how should such cases be dealt with when they do arise? There will always be some cases in which all reasonable attempts are unsuccessful, maybe obviously as in the second case, or less obviously where we simply do not hear back from a person after we have asked them to communicate with their family. It is these cases that we should use to study the challenging issues of respect for confidentiality and right to know about relevant personal health information.

Anneke Lucassen, Michael Parker Wessex Clinical Genetics Service, University of Southampton, Coxford Road, Southampton, UK; Oxford genetic knowledge park, The Ethox Centre, Oxford, UK

Correspondence to: Anneke Lucassen, Wessex Clinical Genetics Service, University of Southampton, Coxford Road, Southampton, UK. Tel.: +44238079 6841;

Fax: 44238079 4346; E-mail: a.m.lucassen@soton.ac.uk

\author{
Reference \\ 1 Lucassen A, Parker M: Confidentiality and serious harm in \\ genetics-preserving the confidentiality of one patient and \\ preventing harm to relatives. EJHG 2004; 12: 93-97.
}

